\title{
Application of the Cylindrical Wave Approach to the Simulation of Buried Utilities
}

\author{
Fabrizio Frezza, ${ }^{1}$ Lara Pajewski, ${ }^{2}$ Cristina Ponti, ${ }^{2}$ and Giuseppe Schettini ${ }^{2}$ \\ ${ }^{1}$ Department of Information Engineering, Electronics and Telecommunications, Sapienza, University of Rome, Via Eudossiana 18, \\ 00184 Roma, Italy \\ ${ }^{2}$ Department of Applied Electronics, Roma Tre University, Via della Vasca Navale 84, 00146 Roma, Italy
}

Correspondence should be addressed to Lara Pajewski, pajewski@uniroma3.it

Received 16 February 2011; Accepted 4 May 2011

Academic Editor: Francesco Soldovieri

Copyright () 2011 Fabrizio Frezza et al. This is an open access article distributed under the Creative Commons Attribution License, which permits unrestricted use, distribution, and reproduction in any medium, provided the original work is properly cited.

The cylindrical wave approach is resumed. It is a full-wave technique for the solution of the two-dimensional plane-wave scattering problem by a set of perfectly conducting and dielectric cylinders buried in a dielectric half-space, or in a finite-thickness slab. This method can be applied for simulating scenarios with cylindrical inhomogeneities buried in the earth. New numerical results are presented, concerning the simulation of buried utilities. In particular, metallic pipes and air cavities are simulated.

\section{Introduction}

The Ground Penetrating Radar (GPR) [1,2] is an established and routine method for the inspection of civil engineering structures. It can provide high-resolution images of the subsurface, typically from 0 to $10 \mathrm{~m}$ depth, through wideband nonsinusoidal electromagnetic waves. The employed frequencies range from $10 \mathrm{MHz}$ to $4 \mathrm{GHz}$. This technique is effective, rapid, nondestructive and non-invasive. It is employed for surveying of roads and highway pavements [3], bridges [4], tunnels [5], and for detecting subsurface cavities and voids [6]. It can also be used for utility sensing [7], for example, to map all the buried structures in a region, enabling rapid installation of a new plant with the minimum disruption and damage to the existing one. Gas, water, sewage, electricity, telephone and cable utilities can be localized. Moreover, the GPR can be used to perform detailed quality controls of reinforced concrete $[8,9]$. An analysis of geological structures can be performed, for the mapping of soil, rock or fill layers in geotechnical investigations and for foundation design [10].

An important factor, among those limiting the GPR surveying of buried pipes and cables, is the density of plant in urban areas: if a lot of underground infrastructures are present, it is difficult to interpret the measured data and to clearly image the scenario, but this is just the situation where clarity is more needed.

The majority of buried plant is within $0.5 \mathrm{~m}$ to $2 \mathrm{~m}$ of the ground surface and it may have a wide variation in its size, may be metallic or nonmetallic, in close proximity to other plant. It may be buried in a wide range of soil types, involving large differences in electromagnetic propagation velocity and absorption. Therefore, obtaining adequate penetration of the emitted radiation together with good resolution is not straightforward, and some compromise has to be accepted.

The block diagram of a radar system is shown in Figure 1. A few nanosecond short impulse of electromagnetic energy is launched by a transmitting antenna. The antenna is mounted on a mobile trolley that is moved forward over the soil, at a very close distance from the ground surface. The energy scattered by the target is gathered by the receiving antenna, which is usually identical to the transmitting antenna and then processed by the receiver, to display the signal in a suitable form for the operator.

Radars solve inverse problems, to estimate the electromagnetic properties of a target, or of a complex scenario, from field measurements. At present, different algorithms are employed in the post-processing of collected GPR data; most of them need a fast and accurate forward-scattering solver, to perform repeated evaluations of the scattered 


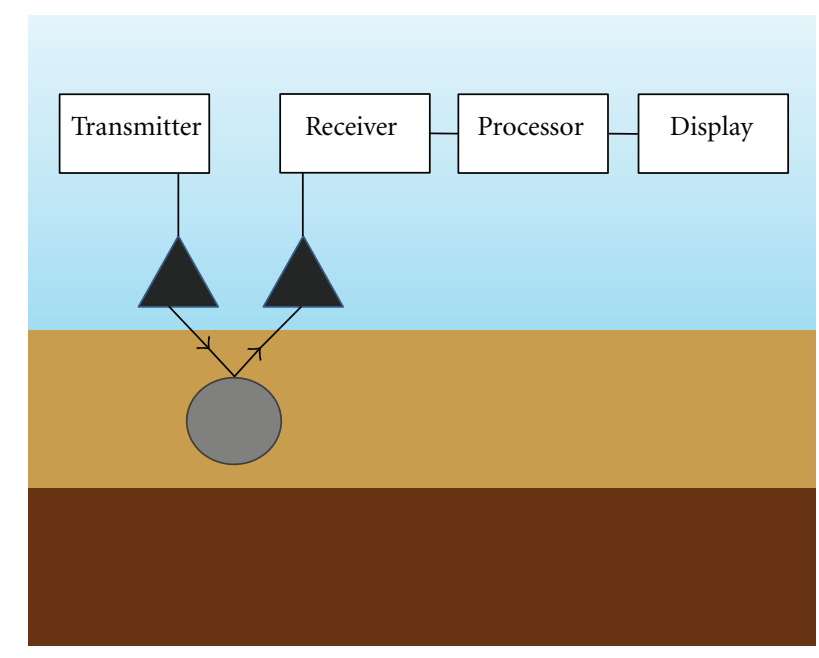

Figure 1: Block diagram of a GPR system.

electromagnetic field due to known targets and to be used in combination with some optimization techniques.

Almost all the objects sought in utility detection are long and thin, so in these applications two-dimensional scattering techniques are employed in the post-processing, being more effective and faster than three-dimensional ones.

The direct two-dimensional electromagnetic-scattering problem of buried objects is, therefore, a theme of great interest in GPR context, and it has been faced by several authors [1], both from a theoretical and a numerical point of view. Different methods have been developed for its solution, both in the frequency and time domains [11-13] (and references therein). Due to the complexity of the problem, many methods present limitations; for example, in [14] the obstacle size and the distance between buried scatterer and air-ground interface have to be much larger than the wavelength, the method proposed in [15] is valid only for small objects, no more than one cylinder is considered and only far-field results are calculated in [16]; some techniques can be applied only when the dielectric contrast between the obstacle and the hosting medium is low $[17,18]$; some approaches suppose the ground to be lossless [11]. The finite-element method (FEM) [19], the finite-difference time-domain technique (FDTD) [20], and the method of moments (MoM) [21] can treat more general configurations and are often used.

The cylindrical-wave approach (CWA) [11-13, 22, 23] is an efficient spectral-domain technique, developed for the rigorous solution of the two-dimensional electromagnetic forward-scattering problem by a finite set of perfectly conducting or dielectric targets, buried in a dielectric halfspace or in a finite-thickness slab. In this method, the field scattered by underground objects is represented in terms of a superposition of cylindrical waves. Use is made of the planewave spectrum [24] to take into account the interaction of such waves with the planar interfaces between air and soil and between different layers in the ground. Suitable reflected [25] and transmitted [11] cylindrical functions are defined. Adaptive integration procedures of Gaussian type, together with acceleration algorithms, are employed for the numerical solution of the relevant spectral integrals [12, 26, 27]. All the multiple-reflection and-diffraction phenomena are taken into account.

The CWA may deal with both TM and TE polarization fields. It can be applied for arbitrary values of permittivity, size, and position of the targets. Obstacles of general shape can be simulated, by means of a suitable set of small circularsection cylinders. Since the CWA is implemented in the frequency domain, dispersive soils can be modelled. The technique has been extended to study the scattering of an incident pulsed wave, with a rather general time-domain shape, a sampling of the incident-field spectrum can be performed, and the scattering problem can be solved in the frequency domain by using the CWA $[13,28]$. The method is accurate and fast; therefore, it may be exploited in iterative algorithms for the solution of inverse problems.

In Section 2, we briefly resume the theoretical basis of the CWA. In Section 3, new results are presented, showing the effectiveness of the method for the sensing of cylindrical inhomogeneities buried in the earth. In particular, an electromagnetic simulation is performed of suitable scenarios in the context of civil engineering applications.

\section{Theoretical Basis of the CWA}

2.1. Perfectly Conducting Cylinders in a Dielectric HalfSpace. The application of the CWA to the solution of the monochromatic plane wave-scattering problem by a finite set of perfectly conducting cylinders buried in a dielectric halfspace is described in detail in [11]. In this subsection, the method is briefly resumed.

Let us consider $N$ perfectly conducting circular cylinders with possibly different radii, buried in a linear, isotropic, homogeneous, dielectric, lossless half-space, as schematized in Figure 2. Each cylinder is parallel to the $y$ axis; the structure is assumed to be infinite along $y$ direction. A monochromatic plane wave, with wavevector $k^{i}$ lying in the $x z$ plane, impinges at an angle $\varphi_{\mathrm{i}}$ from medium 0 (a vacuum) on the planar interface with medium 1 . We introduce a normalized reference frame $(O, \xi, \zeta)$, with coordinates $\xi=$ $k_{0} x$ and $\zeta=k_{0} z, k_{0}=2 \pi / \lambda_{0}$ being the vacuum wavenumber and $\lambda_{0}$ the vacuum wavelength. In such reference frame, the $q$ th cylinder has axis located in $\left(\chi_{q}, \eta_{q}\right)$, and its dimensionless radius is called $\alpha_{q}=k_{0} a_{q}$. The time dependence of the field is assumed to be $e^{-i \omega t}$, where $\omega$ is the angular frequency. The solution to the scattering problem is carried out in terms of $V(\xi, \zeta)$, representing the $y$-component of the electric/magnetic field: $V=E_{y}(\xi, \zeta)$ for $\mathrm{TM}^{(y)}$ polarization, and $V=H_{y}(\xi, \zeta)$ for $\mathrm{TE}^{(y)}$ polarization.

In order to obtain a rigorous solution for $V(\xi, \zeta)$, the total field is expressed as the superposition of a set of terms, produced by the interaction between the incident field, the interface and the cylinders: the incident plane wave, the reflected field (due to the reflection in medium 0 of the incident plane wave by the interface), and the transmitted field (due to the transmission in medium 1 of the incident plane wave by the interface); the field scattered 


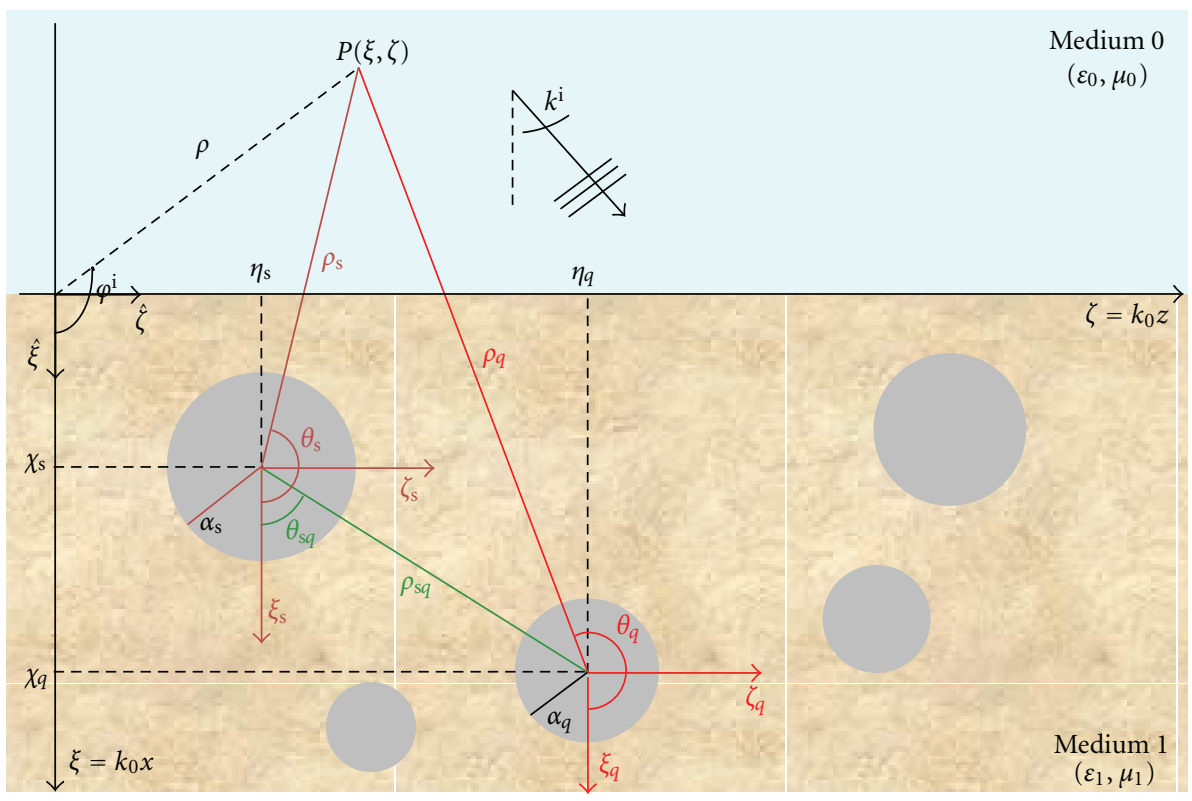

FIGURE 2: Geometry of the scattering problem, with cylinders buried in a dielectric half-space.

by the cylinders in medium 1 , the scattered-reflected field (due to the reflection in medium 1 of the scattered field by the interface), the scattered-transmitted field (due to the transmission in medium 0 of the scattered field by the interface).

The scattered field is expressed as the sum of the fields scattered by each buried obstacle. The field scattered by a cylinder is represented in terms of a superposition of cylindrical waves, by means of unknown coefficients, and use is made of the plane-wave spectrum of such waves [24] to treat their reflection and transmission by the interface. The presence of the planar interface, in fact, is taken into account by means of its complex plane-wave reflection and transmission coefficients. The final expression for the scattered field turns out to be, in a reference system centered on the $q$ th cylinder axis $(q=1, \ldots, N)$,

$$
\begin{aligned}
V_{s} & =V_{0} \sum_{m=-\infty}^{+\infty} J_{m}\left(n_{1} \rho_{q}\right) e^{i m \theta_{q}} \sum_{h=1}^{N} \sum_{l=-\infty}^{+\infty} i^{l} e^{i l \varphi_{t}} C_{h l} \\
& \times\left[C W_{l-m}\left(n_{1} \xi_{h q}, n_{1} \zeta_{h q}\right)\left(1-\delta_{h q}\right)+\frac{H_{m}^{(1)}\left(n_{1} \rho_{q}\right)}{J_{m}\left(n_{1} \rho_{q}\right)} \delta_{h q} \delta_{m l}\right],
\end{aligned}
$$

where $V_{0}$ is the incident plane-wave amplitude, $J_{m}$ is the firstkind Bessel function of integer order $m, n_{1}$ is the refractive index of the medium hosting the scatterers, $\varphi_{t}$ is the angle of the transmitted plane wave (according to Snell's law), $c_{h e}$ are unknown coefficients, $C W_{\ell}(\xi, \zeta)=H_{\ell}^{(1)}(\rho) e^{i \ell \theta}$ is the cylindrical wave, and $H_{\ell}^{(1)}$ is the first-kind Hankel function of integer order $\ell$; moreover, $\xi_{h q}$ and $\zeta_{h q}$ are the distances, along $\xi$ and $\zeta$ directions, of the axes of the $h$ th and $q$ th cylinders; finally, $\delta$ is the Kronecker symbol.

To express the scattered-reflected and scatteredtransmitted fields, the presence of the planar surface is taken into account by considering the reflection and transmission of each elementary plane wave constituting the Fourier spectrum of a cylindrical function. The scattered-reflected field is given by the sum of the fields scattered by each buried cylinder and reflected by the interface; by introducing the reflected cylindrical functions $R W_{\ell}[11]$, the final expression is

$$
\begin{aligned}
V_{\text {sr }}= & V_{0} \sum_{m=-\infty}^{+\infty} J_{m}\left(n_{1} \rho_{q}\right) e^{i m \theta_{q}} \sum_{h=1}^{N} \sum_{l=-\infty}^{+\infty} C_{h l} i^{l} e^{-i l \varphi_{t}} \\
& \times R W_{m+l}\left[-n_{1}\left(\chi_{h}+\chi_{q}\right), n_{1}\left(\eta_{q}-\eta_{h}\right)\right] .
\end{aligned}
$$

The scattered-transmitted field is given by the sum of the fields scattered by each buried cylinder and transmitted to medium 0 . Introducing the transmitted cylindrical functions $T W_{\ell}[11]$, it can be found that

$$
V_{\text {st }}=V_{0} \sum_{h=1}^{N} c_{h l} i^{l} e^{-i l \varphi_{t}} T W_{l}\left(\xi_{h}, \zeta_{h}, \chi_{h}\right),
$$

Given the expressions of all the field terms, the boundary conditions on the cylinder surfaces are imposed; due to the hypothesis of perfectly conducting cylinders, the tangential component of the total electric field must vanish. A linear system for the unknown coefficients of the cylindrical wave expansions is obtained. Once such system is solved, the $V(\xi, \zeta)$ field is determined in any point of the space and for both polarizations. It is possible to derive all the other components of the electromagnetic field by using Maxwell's equations.

2.2. Dielectric Cylinders in a Dielectric Half-Space. When the buried scatterers are dielectric cylinders, the field is present also inside them. This problem is treated in detail in [12]. The expression of the field internal to the $q$ th cylinder is given 


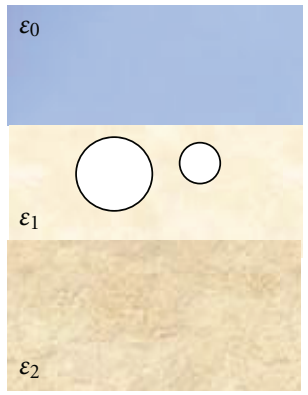

(a)

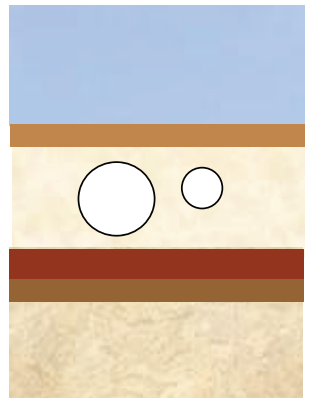

(d)

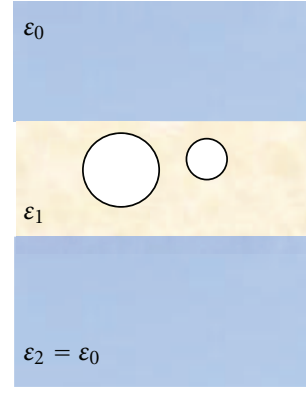

(b)

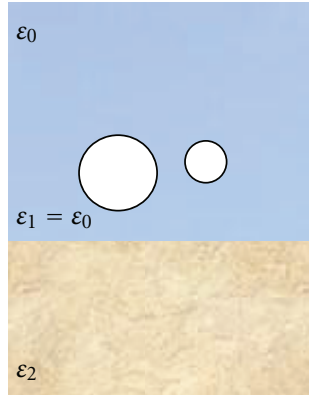

(e)

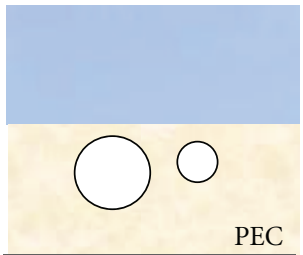

(c)

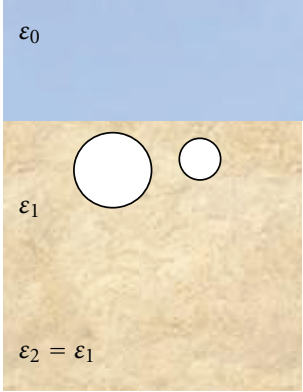

(f)

FIgURE 3: Cylinders in a finite-thickness slab, various scenarios.

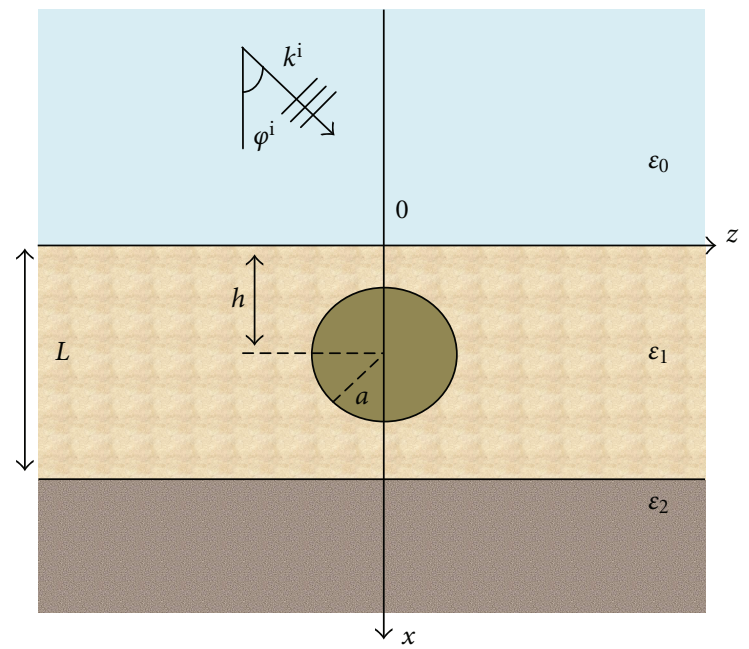

Figure 4: Geometrical layout of a buried utility.

by an expansion in terms of first-kind Bessel functions with unknown coefficients $d_{q m}$, as follows:

$$
V_{\mathrm{cq}}=V_{0} \sum_{m=-\infty}^{+\infty} d_{q m} i^{m} e^{-i m \varphi_{t}} J_{m}\left(n_{c q} \rho_{q}\right) e^{i m \theta_{q}}
$$

where $n_{c q}$ is the refractive index of the cylinder.

On the cylinder surfaces, the boundary conditions have to be imposed. A linear system is thus obtained, for the unknown coefficients of the cylindrical wave expansions and for the $d_{q m}$ coefficients. Once the system has been solved, the total electromagnetic field is completely determined in any point of the space.

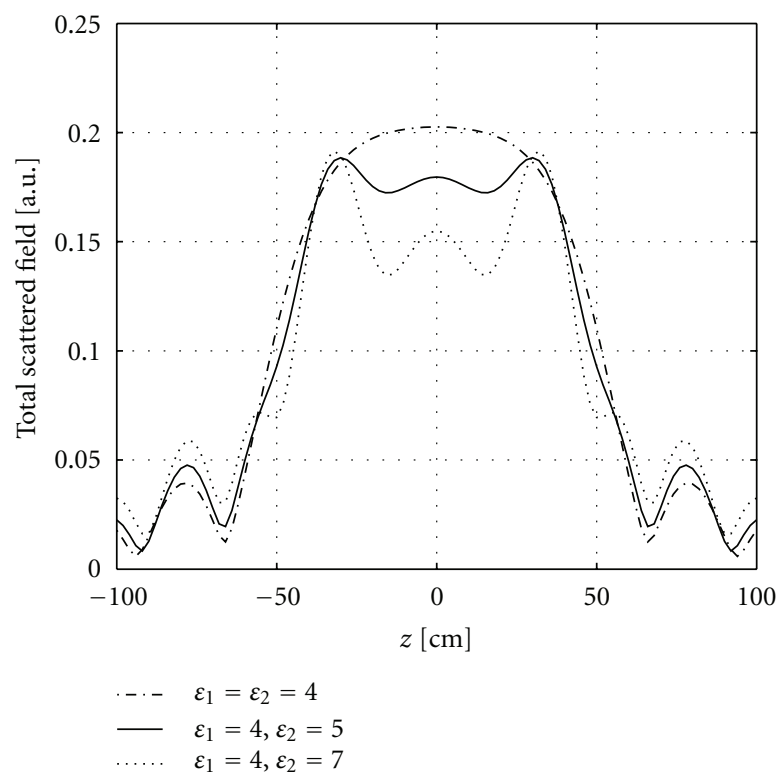

FIgURE 5: Total scattered field in air, along a line parallel to the interface, for the layout of Figure 4, with $L=120 \mathrm{~cm}$.

2.3. Cylinders in a Finite-Thickness Slab. When the scatterers are inside a finite-thickness slab, among two different halfspaces, additional reflected-transmitted, multiple-reflected and multiple-reflected-transmitted cylindrical functions are introduced, to consider the complicated interaction between the cylinders and the planar interfaces delimiting the slab. The case of perfectly conducting cylinders is solved in [22]; the solution to the scattering problem by dielectric cylinders in a slab is described in [23]. 


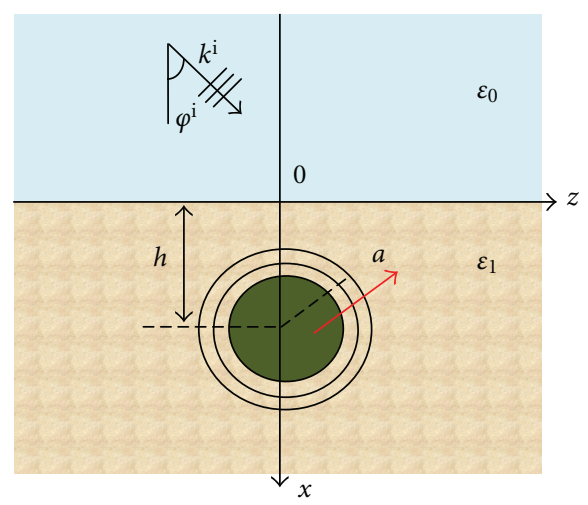

(a)

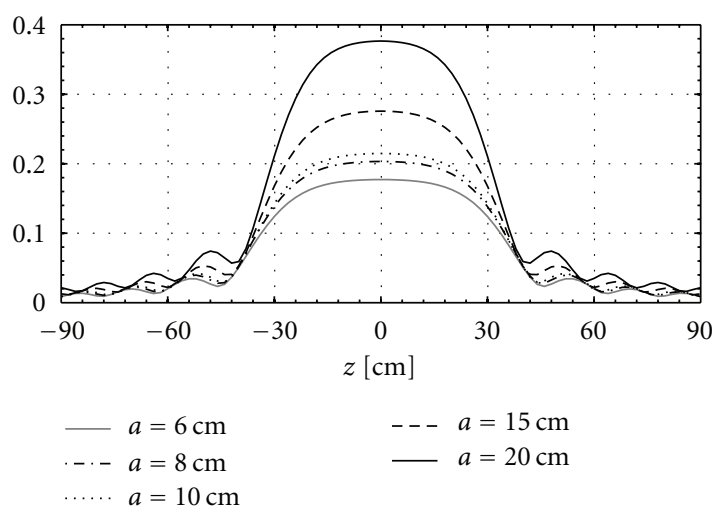

(b)

Figure 6: Total scattered field in air, along a line parallel to the interface, for different values of the radius of the metallic utility.

It is important to observe that this extension of the method allows to simulate several interesting scenarios, as sketched in Figure 3: objects in a soil layer, above a ground of different permittivity (a), objects inside a slab among two identical half-spaces, for example, cylinders in a wall (b), or else scatterers in a slab terminated on a perfectly conducting surface (c), in a layer of a stratified medium (d), or, finally, cylinders above (e) or in (f) a dielectric half-space.

2.4. Pulsed-Wave Analysis. By using the CWA technique, it is possible to solve the scattering problem by buried perfectly conducting or dielectric cylinders of an incident pulsed plane wave, with a rather general time-domain shape. We have to perform a sampling of the incident-field spectrum and of the spectra of the various field terms involved (reflected, transmitted, scattered, scattered-reflected, scattered-transmitted, etc.). The scattering problem can be solved in the frequency domain for any sample through the CWA. Finally, by means of an inverse transform, the solution in the time domain can be calculated. This procedure is described in detail in $[13,28]$.

\section{Numerical Results}

Let us consider an underground metallic utility at a standard burial depth of $50 \mathrm{~cm}$ : this suggests to operate in a frequency

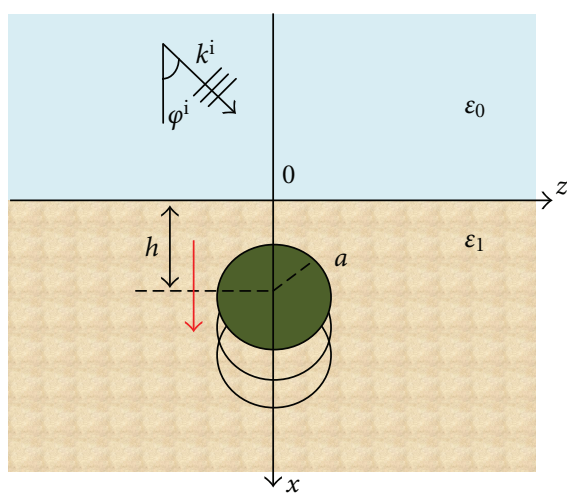

(a)

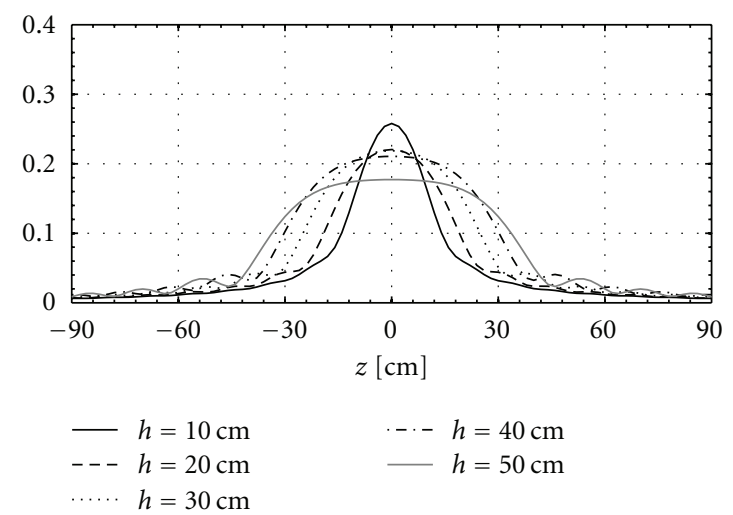

(b)

FIgURE 7: Total scattered field in air, along a line parallel to the interface, for different values of the burial depth.

range between 1 and $1.5 \mathrm{GHz}$. A circular section with a $6 \mathrm{~cm}$ diameter is assumed for a target, embedded in soil layer of relative permittivity $\varepsilon_{1}=4$. In the CWA analysis with normalized quantities, these geometrical values correspond to a depth $\chi=5 \pi$ and a radius $\alpha=0.6 \pi$, at a frequency of $1.5 \mathrm{GHz}$. The layout is sketched in Figure 4. The total scattered field is evaluated in the upper medium, at the nearfield distance of $5 \mathrm{~cm}$, for a normally incident plane wave in TM polarization (electric field parallel to the cylinder axis).

In Figure 5, the scattered field is plotted for a layer of thickness $L=120 \mathrm{~cm}$ and for different permittivity values of the lower half-space. The relative permittivity of dry soil, such as dry sand, clay, and rock, is assumed as being comprised between 4 and 7. The hypothesis of dry materials is made to better meet the approximation of lossless materials of our analysis; moreover, in practical surveys the application of GPR to utility detection is limited by attenuation due to wet soil. When $\varepsilon_{1}=\varepsilon_{2}=4$, the scatterer is buried in a semiinfinite medium. The scattered-field shows a behaviour, along a line parallel to the interface, that strongly depends on the presence of the second medium: when the object is in a finite-thickness slab, three peaks can be appreciated in the main lobe of the scattered field, while there are not oscillations in the main lobe when the cylinder is buried in a semi-infinite medium. 


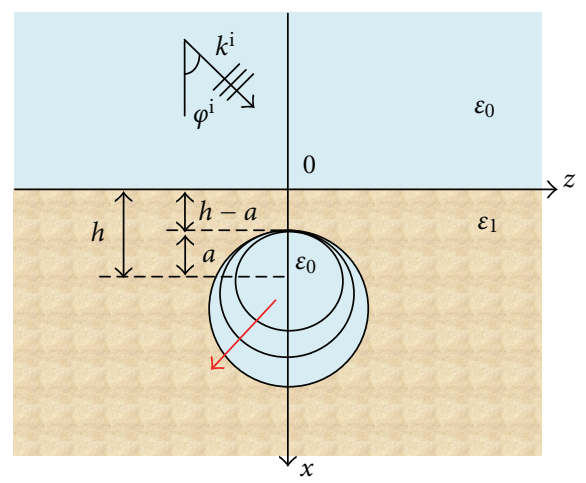

(a)

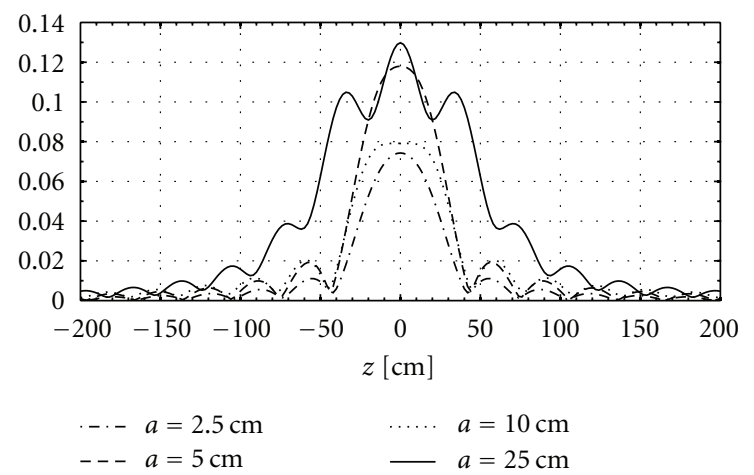

(b)

Figure 8: Total scattered field in air, along a line parallel to the interface, for air cavities of different size.

In Figures 6 and 7, the metallic utility is buried in a dielectric half-space with $\varepsilon_{1}=4$. In particular, in Figure 6 different values of the cylinder radius are considered, and of course for a bigger utility, a stronger scattered field is observed. In Figure 7, an electric utility with radius $a=6 \mathrm{~cm}$ is buried at different depths; it can be observed that when it is deeper, the scattered field is less directive; since the soil is considered lossless, we do not obtain a significant reduction of the scattered field amplitude.

Let us now consider an underground air cavity in a dielectric half-space of permittivity $\varepsilon_{1}=4$. As in previous simulations, the frequency is $1.5 \mathrm{GHz}$, and the total scattered field is evaluated in the air, at the near-field distance of $5 \mathrm{~cm}$, for a normally incident plane wave in TM polarization. In Figure 8, different values of the cavity radius are considered, the distance between air-soil interface and the target is fixed and it is $h-a=10 \mathrm{~cm}$; the electromagnetic effect of a bigger cavity is stronger and the main beam of the scattered field has a more jagged behaviour. In Figure 9 the radius is fixed, $a=10 \mathrm{~cm}$, and different values of the burial depth are simulated; considerations similar to those of Figure 7 apply.

We finally present, in Figure 10, time-domain results for three buried metallic utilities. The geometry is sketched in the figure. The cylinder radii are $a_{1}=a_{3}=2 \mathrm{~cm}, a_{2}=4 \mathrm{~cm}$, the burial depths are $h_{1}=30 \mathrm{~cm}, h_{2}=40 \mathrm{~cm}, h_{3}=35 \mathrm{~cm}$, and the distances along $z$ axis are $z_{1}=0, z_{2}=21 \mathrm{~cm}$ and

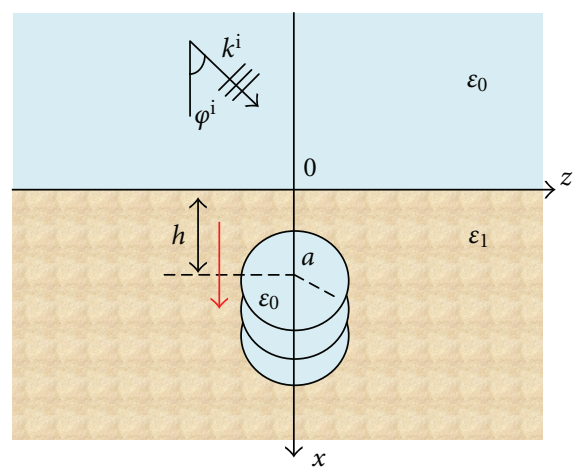

(a)

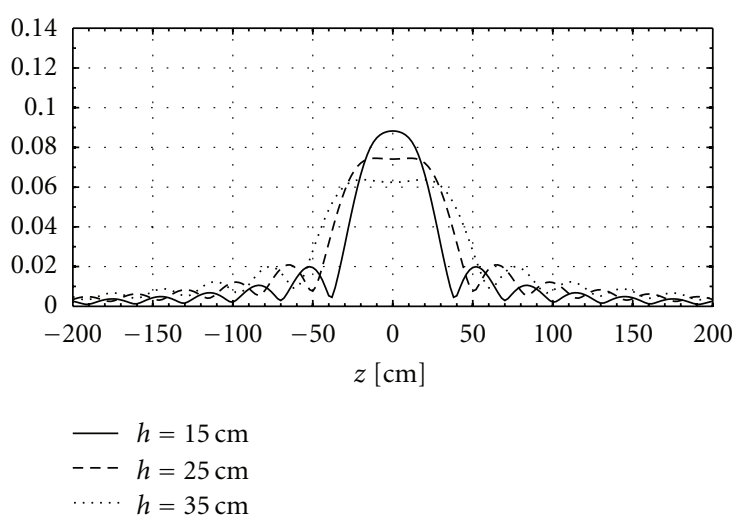

(b)

Figure 9: Total scattered field in air, along a line parallel to the interface, for air cavities located at various depths.

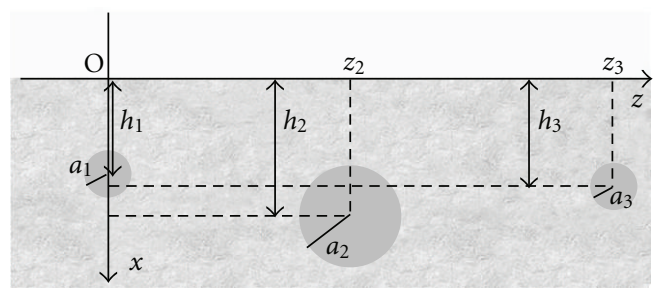

(a)

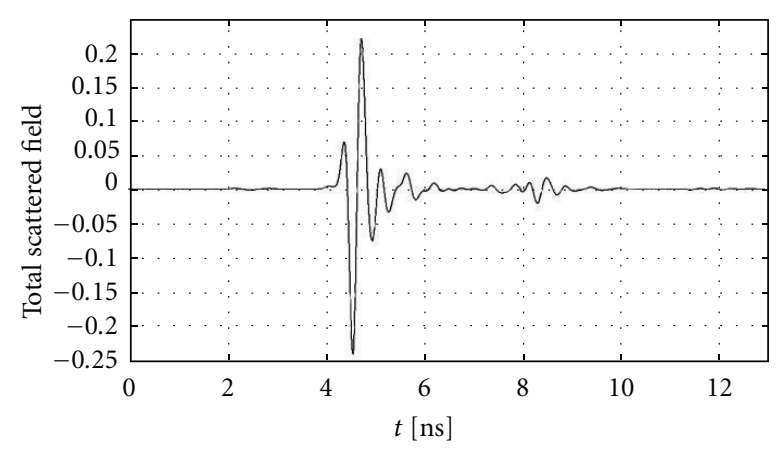

(b)

FIgURE 10: Time-domain results for three buried metallic utilities. 
$z_{3}=42 \mathrm{~cm}$. The polarization of the incident field is TM, the frequency is $1.433 \mathrm{GHz}$, and the time-domain shape is the one proposed in [13]. The observation point is $15 \mathrm{~cm}$ above the ground, aligned with the bigger cylinder axis. We plotted the early-time response of the structure, that is, the total scattered field as a function of time, over a time interval that is of the order of the incident pulse duration and its propagation from the observation point to the targets. Several wavefronts can be identified due to combined effects of the diffraction from the cylinders, the reverberations between the cylinders and the air-ground interface, the creeping-wave circumnavigation of the cylinders.

\section{Conclusions}

In this paper, our work on the development of the cylindrical-wave approach for the solution of the twodimensional plane wave scattering problem by a set of perfectly conducting and dielectric cylinders buried in a dielectric half-space or in a finite-thickness slab, is shortly reviewed. The proposed full-wave technique deals with both TM and TE polarization cases and yields results in both the near- and the far-field zones. Moreover, it may be applied for any value of the scatterers size and of the distance between the obstacles and the interface between air and soil.

The method can be used for the characterization of suitable scenarios in the context of ground-penetrating radar (GPR) applications, which usually employ purelynumerical finite-difference (FD) techniques. The CWA could be exploited in iterative algorithms for the solution of inverse problems, where fast, efficient, and accurate forward solvers are needed to perform repeated evaluations. The approach can be employed to study the scattering of an incident pulsed plane wave, with a rather general time-domain shape.

We have presented new numerical results concerning the simulation of buried utilities. In particular, metallic pipes and air cavities have been considered, and the effectiveness of the method for the sensing of cylindrical inhomogeneities buried in the earth has been shown.

\section{References}

[1] D. J. Daniels, Ground Penetrating Radar, IEE, London, UK, 2nd edition, 2004.

[2] H. Jol, Ground Penetrating Radar: Theory and Applications, Elsevier, Amsterdam, The Netherlands, 2009.

[3] A. Benedetto and S. Pensa, "Indirect diagnosis of pavement structural damages using surface GPR reflection techniques," Journal of Applied Geophysics, vol. 62, no. 2, pp. 107-123, 2007.

[4] J. Hugenschmidt, "Accuracy and reliability of radar results on bridge decks," in Proceedings of the 10th International Conference on Ground Penetrating Radar, pp. 371-374, Delft, The Netherlands, June 2004.

[5] M. Kuloglu and C.-C. Chen, "Ground penetrating radar for tunnel detection," in Proceedings of the International Geoscience and Remote Sensing Symposium (IGARSS '10), pp. 4314-4317, Honolulu, Hawaii, USA, July 2010.

[6] T. Mochales, A. M. Casas, E. L. Pueyo et al., "Detection of underground cavities by combining gravity, magnetic and ground penetrating radar surveys: a case study from the
Zaragoza area, NE Spain," Environmental Geology, vol. 53, no. 5, pp. 1067-1077, 2008.

[7] A. Simi, G. Manacorda, M. Miniati, S. Bracciali, and A. Buonaccorsi, "Underground asset mapping with dualfrequency dual-polarized GPR massive array," in Proceedings of the 13th International Conference on Ground Penetrating Radar, p. 5, Lecce, Italy, June 2010.

[8] W. L. Lai, T. Kind, and H. Wiggenhauser, "Detection of accelerated reinforcement corrosion in concrete by ground penetrating radar," in Proceedings of the 13th International Conference on Ground Penetrating Radar, p. 4, Lecce, Italy, June 2010.

[9] B. Filali, F. Boone, J. Rhazi, and G. Ballivy, "Design and calibration of a large open-ended coaxial probe for the measurement of the dielectric properties of concrete," IEEE Transactions on Microwave Theory and Techniques, vol. 56, no. 10, pp. 2322-2328, 2008.

[10] M. Tallini, A. Giamberardino, D. Ranalli, and M. Scozzafava, "GPR survey for investigation in building foundations," in Proceedings of the 10th International Conference on Ground Penetrating Radar, pp. 395-397, Delft, The Netherlands, June 2004.

[11] M. Di Vico, F. Frezza, L. Pajewski, and G. Schettini, "Scattering by a finite set of perfectly conducting cylinders buried in a dielectric half-space: a spectral-domain solution," IEEE Transactions on Antennas and Propagation, vol. 53, no. 2, pp. 719-727, 2005.

[12] M. Di Vico, F. Frezza, L. Pajewski, and G. Schettini, "Scattering by buried dielectric cylindrical structures," Radio Science, vol. 40, no. 6, Article ID RS6S18, 11 pages, 2005.

[13] F. Frezza, P. Martinelli, L. Pajewski, and G. Schettini, "Shortpulse electromagnetic scattering by buried perfectly conducting cylinders," IEEE Geoscience and Remote Sensing Letters, vol. 4, no. 4, pp. 611-615, 2007.

[14] K. Hongo and A. Hamamura, "Asymptotic solutions for the scattered field of plane wave by a cylindrical obstacle buried in a dielectric half-space," IEEE Transactions on Antennas and Propagation, vol. AP-34, no. 11, pp. 1306-1312, 1986.

[15] J. D. Kanellopoulos and N. E. Buris, "Scattering from conducting cylinders embedded in a lossy medium," International Journal of Electronics, vol. 57, no. 3, pp. 391-401, 1984.

[16] D. E. Lawrence and K. Sarabandi, "Electromagnetic scattering from a dielectric cylinder buried beneath a slightly rough surface," IEEE Transactions on Antennas and Propagation, vol. 50, no. 10, pp. 1368-1376, 2002.

[17] D. A. Hill, "Electromagnetic scattering by buried objects of low contrast," IEEE Transactions on Geoscience and Remote Sensing, vol. 26, no. 2, pp. 195-203, 1988.

[18] D. A. Hill, "Near-field detection of buried dielectric objects," IEEE Transactions on Geoscience and Remote Sensing, vol. 27, no. 4, pp. 364-368, 1989.

[19] K. O.'Neill, S. A. Haider, S. D. Geimer, and K. D. Paulsen, "Effects of the ground surface on polarimetric features of broadband radar scattering from subsurface metallic objects," IEEE Transactions on Geoscience and Remote Sensing, vol. 39, no. 7, pp. 1556-1565, 2001.

[20] K. Demarest, R. Plumb, and Z. Huang, "FDTD modeling of scatterers in stratified media," IEEE Transactions on Antennas and Propagation, vol. 43, no. 10, pp. 1164-1168, 1995.

[21] S. Vitebskij, K. Sturgess, and L. Carin, "Short-pulse planewave scattering from buried perfectly conducting bodies of revolution," IEEE Transactions on Antennas and Propagation, vol. 44, no. 2, pp. 143-151, 1996. 
[22] F. Frezza, L. Pajewski, C. Ponti, and G. Schettini, "Scattering by perfectly conducting circular cylinders buried in a dielectric slab through the cylindrical wave approach," IEEE Transactions on Antennas and Propagation, vol. 57, no. 4, pp. 1208-1217, 2009.

[23] F. Frezza, L. Pajewski, C. Ponti, and G. Schettini, "Scattering by dielectric circular cylinders in a dielectric slab," Journal of the Optical Society of America A, vol. 27, no. 4, pp. 687-695, 2010.

[24] G. Cincotti, F. Gori, M. Santarsiero, F. Frezza, F. Furnò, and G. Schettini, "Plane wave expansion of cylidrical functions," Optics Communications, vol. 95, no. 4-6, pp. 192-198, 1993.

[25] R. Borghi, F. Gori, M. Santarsiero, F. Frezza, and G. Schettini, "Plane-wave scattering by a perfectly conducting circular cylinder near a plane surface: cylindrical-wave approach," Journal of the Optical Society of America A, vol. 13, no. 3, pp. 483-493, 1996.

[26] R. Borghi, F. Frezza, C. Santini, M. Santarsiero, and G. Schettini, "Numerical study of the reflection of cylindrical waves of arbitrary order by a generic planar interface," Journal of Electromagnetic Waves and Applications, vol. 13, no. 1, pp. 27-50, 1999.

[27] R. Borghi, F. Frezza, M. Santarsiero, C. Santini, and G. Schettini, "Quadrature algorithm for the evaluation of a 2D radiation integral with a highly oscillating kernel," Journal of Electromagnetic Waves and Applications, vol. 14, no. 10, pp. 1353-1370, 2000.

[28] F. Frezza, P. Martinelli, L. Pajewski, and G. Schettini, "A CWAbased detection procedure of a perfectly-conducting cylinder buried in a dielectric half-space," Progress In Electromagnetics Research B, vol. 7, pp. 265-280, 2008. 

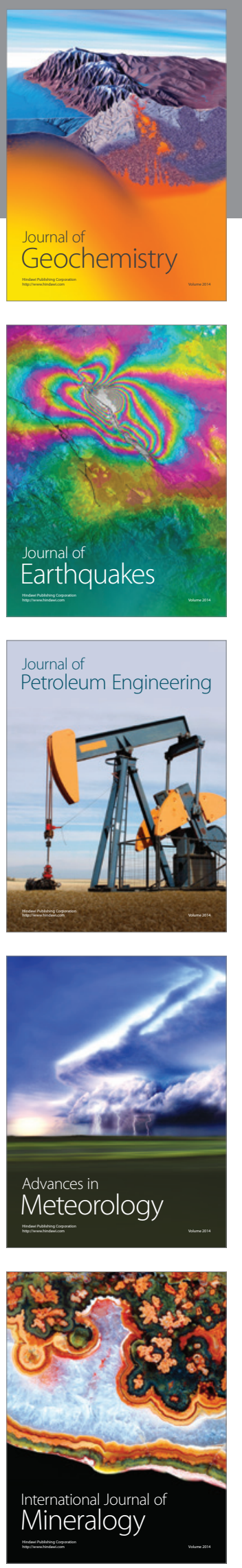
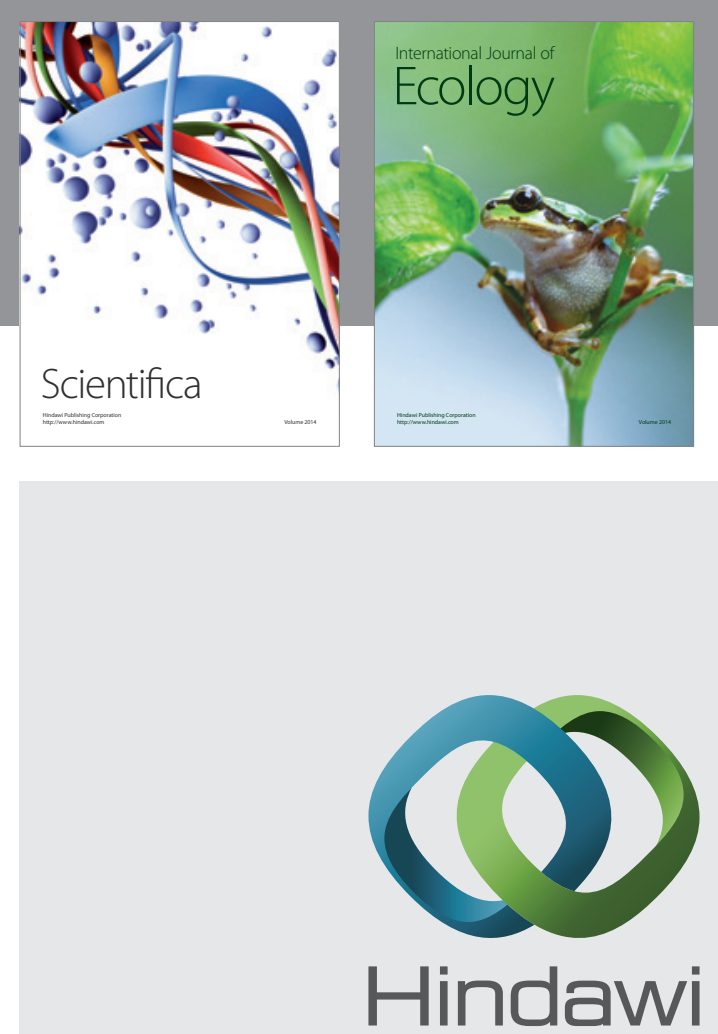

Submit your manuscripts at http://www.hindawi.com
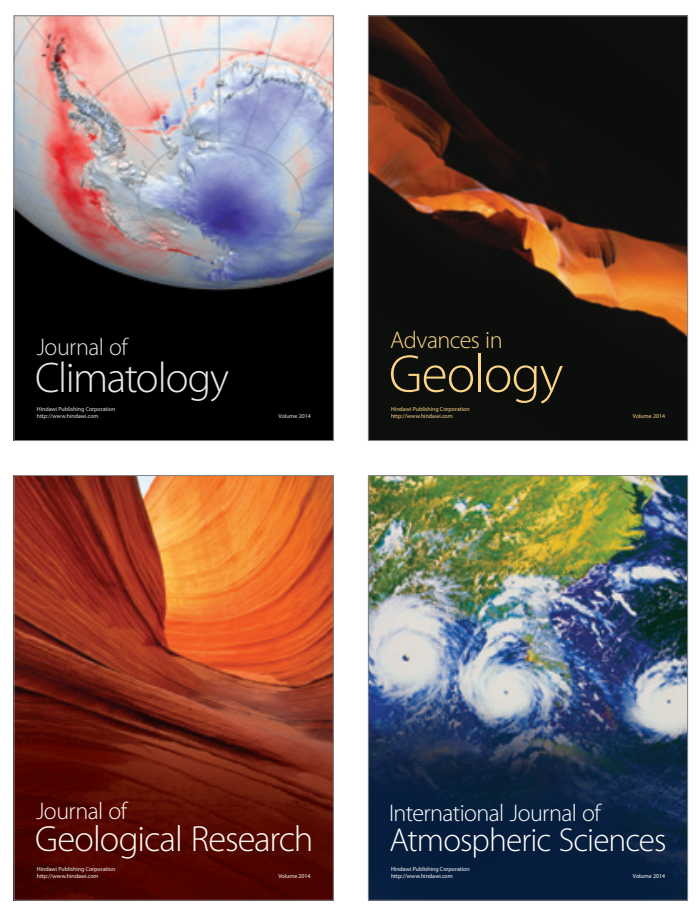
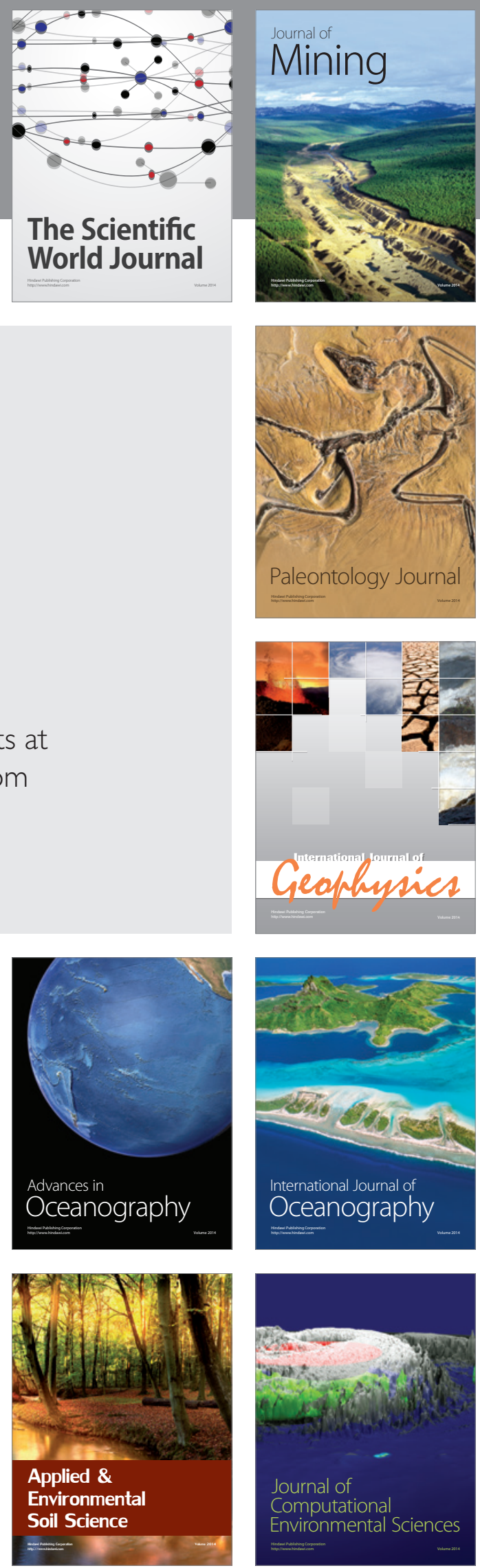